\title{
Evaluation of the relationship between splenic iron overload and liver, heart and muscle features evident on $\mathrm{T} 2$-weighted magnetic resonance imaging
}

\author{
Mehmet Güli Çetinçakmak ${ }^{1, A-E}$, Salih Hattapoğlu, $1, \mathrm{~A}, \mathrm{~B}, \mathrm{D}$, Murat Söker ${ }^{2, B, C}$, \\ Faysal Ekici' ${ }^{1, A, B, D}$, Kamil Yilmaz, ${ }^{2, B C}$, Cemil Göya ${ }^{1, A-C}$, Cihad Hamidi ${ }^{1, A, D-F}$ \\ ${ }^{1}$ Department of Radiology, Medical School, Dicle University, Diyarbakir, Turkey \\ 2 Department of Pediatrics, Medical School, Dicle University, Diyarbakir, Turkey \\ A - research concept and design; $\mathrm{B}$ - collection and/or assembly of data; $\mathrm{C}$ - data analysis and interpretation; \\ $D$ - writing the article; $E$ - critical revision of the article; $F$ - final approval of the article
}

Address for correspondence

Mehmet Güli Çetinçakmak

E-mail: drmehmetguli@gmail.com

Funding sources

None declared

Conflict of interest

None declared

Received on September 22, 2017

Reviewed on April 16, 2018

Accepted on January 21, 2020

Published online on May 5, 2020

\section{Cite as}

Güli Çetinçakmak MG, Hattapoğlu S, Söker M, et al. Evaluation of the relationship between splenic iron overload and liver, heart and muscle features evident on $\mathrm{T} 2^{*}$-weighted magnetic resonance imaging. Adv Clin Exp Med. 2020;29(4):475-480. doi:10.17219/acem/116758

DOI

10.17219/acem/116758

Copyright

Copyright by Author(s)

This is an article distributed under the terms of the

Creative Commons Attribution 3.0 Unported (CC BY 3.0)

(https://creativecommons.org/licenses/by/3.0/)

\begin{abstract}
Background. Splenic iron overload is the most common clinical condition in patients with thalassemia. However, few studies of the effects of splenectomy have been published.

Objectives. To evaluate the relationship between splenic iron overload and liver, heart and muscle features visible in $\mathrm{T}^{*}$-weighted magnetic resonance imaging, and to investigate the effects of splenectomy on these tissues in patients with beta-thalassemia major (TM).

Material and methods. We retrospectively included 131 patients (76 male and 55 female) diagnosed with TM. All radiological assessments were performed with the aid of a Philips Achieva 1.5T scanner running a multiecho gradient-echo sequence. Hepatic and splenic $T 2^{*}$ values were assessed in the same gradient multiecho series. Muscle $T 2^{*}$ values were assessed in the shoulder girdle muscles adjacent to the heart area. The relationships among splenic $T 2^{*}$, hepatic $T 2^{*}$, cardiac $\mathrm{T} 2^{*}$ and muscle $\mathrm{T} 2^{*}$ parameters, serum ferritin levels, age and other parameters were evaluated.

Results. The splenic T2* value correlated with serum ferritin level and the hepatic T2* value $(p<0.001$ and $p<0.001$, respectively). The splenic $2^{*}$ value did not correlate with age, cardiac or muscle $2^{*}$ values, or with spleen size ( $p=0.27,0.21,0.99$, and 0.39 , respectively). The muscle $T 2^{*}$ value correlated weakly with the serum ferritin level $(p=0.022)$. The cardiac $T 2^{*}$ value was lower and the liver size greater in patients who had undergone splenectomy compared with those who had not ( $p<0.001$ and 0.001 , respectively).

Conclusions. Splenic iron overload correlated with hepatic overload and the serum ferritin level. Splenectomy increased cardiac iron overload and triggered liver enlargement. However, the muscle iron overload was low and the muscles were therefore unaffected by splenectomy.
\end{abstract}

Key words: spleen, iron overload, MR imaging, thalassemia 


\section{Introduction}

Beta-thalassemia major (TM) is a hereditary form of hemolytic anemia characterized by impaired globin B-chain output. Patients with TM require red blood cell transfusions every 2 to 3 weeks. In these patients, senescent native and transfusional erythrocytes are eliminated by the Kupffer cells (phagocytic macrophages) of the spleen and liver, and the released iron is subsequently transported to the plasma by ferroportin. ${ }^{1}$ As the total body iron level increases after multiple transfusions and enhanced intestinal absorption, excess iron is stored in the liver and spleen. ${ }^{1,2}$ In thalassemia, the transfusion requirements are increased by spleen hyperactivity, which is often evident in the first decade of life. This condition is termed "hypersplenism" and is treated with splenectomy. ${ }^{3}$

Assessments of tissue iron overload may be either invasive or noninvasive. Biopsies can be used to assess hepatic iron overload, ${ }^{4,5}$ but are compromised by standardization problems and the risk of complications. Magnetic resonance imaging (MRI) is a noninvasive method frequently used to assess tissue iron overload, and is the only option for assessing iron overload in the heart, since cardiac biopsies are more variable and more dangerous than hepatic biopsies. ${ }^{6}$

Many studies have been conducted on cardiac and hepatic iron overload. ${ }^{5,7-10}$ The spleen is the second most common iron deposition site, after the liver. However, in some studies on splenic iron overload, contradictory results have been reported. ${ }^{11-14}$

In the present study, we evaluated the relationships among splenic iron overload and that of the liver, heart and muscles, as well as the effects of splenectomy on these tissues in TM patients using T2\%-weighted MRI.

\section{Material and methods}

\section{Patients}

We retrospectively studied 131 TM patients - 76 (58\%) men and 55 (42\%) women - referred to our clinic between April 2014 and December 2016 for assessments of cardiac and hepatic iron overload using gradient echo (GRE) T2\%weighted MRI. Patients with thalassemia intermedia and primary hemochromatosis were excluded, as were those yielding poor quality MR images. The study was approved by our local ethics committee.

\section{Cardiomuscular and hepatic-splenic examinations}

All radiological assessments were performed using an Achieva 1.5-T scanner (Philips, Amsterdam, the Netherlands). Cardiac and hepatic T2*-weighted sequences were obtained using an RC SENSE-body coil (Philips) with electrocardiographic (ECG) respiratory gating. Multiecho gradient echo sequences were obtained from the central liver
Table 1. Splenic, hepatic, cardiac, and muscle multiecho gradient-echo sequences obtained with MRI parameters

\begin{tabular}{|l|c|c|}
\hline \multicolumn{1}{|c|}{ MRI parameters } & Cardiac and muscle & Liver and spleen \\
\hline Slace thickness & $10 \mathrm{~mm}$ & $10 \mathrm{~mm}$ \\
\hline FOV & $320 \times 320$ & $320 \times 320$ \\
\hline Flip angle & 30 & 45 \\
\hline RT & $16 \mathrm{~ms}$ & $400 \mathrm{~ms}$ \\
\hline Echo times & $7(3,5,7,10,12,14 \mathrm{~ms})$ & $\begin{array}{c}14 \text { (from } 1 \text { to } 14 \mathrm{~ms} \\
\text { with 1 ms increment) }\end{array}$ \\
\hline
\end{tabular}

MRI - magnetic resonance imaging; FOV - field of view; RT - repetition time.

zones with transverse plane for the assessment of hepatic $\mathrm{T} 2 *$ values. A short-axis, midventricular, cardiac-gated multiecho gradient echo sequence was used to derive cardiac T2* values (Table 1 ).

Splenic T2* values were calculated using CMR software (Cardiovascular Imaging Solutions, London, UK). Hepatic and splenic $\mathrm{T} 2 *$ values were calculated using the same echo series. The regions of interest in the spleen and liver covered only tissue. The tissue did not move artifacts outside of vascular and subcapsular spaces. (Fig. 1-2).

The cardiac and muscle $\mathrm{T} 2 *$ values were calculated using the same echo series. Cardiac T2* values were calculated in the mid-ventricular septum (Fig. 3). The muscle T2* values were from shoulder girdle muscles (the subscapular, infrascapular, deltoid, and pectoral muscles) examined in short-axis cardiac sequences (Fig. 4). The calculations were performed using data from regions with no motion artifacts. Within 7 days from the MRI, abdominal ultrasonography (USG) was performed in all the patients; the images were recorded and liver sizes were measured from the midclavicular line of the craniocaudal diameter. Spleen size was taken as the largest diameter on the plane in which the splenic notch was visible. Splenectomy status was recorded. Clinical data including the patients' ages and serum ferritin levels within the previous 2 months was retrieved from their medical records.

\section{Statistical analysis}

All the data was evaluated using SPSS v. 18.0 for Windows (SPSS Inc., Chicago, USA). Only the cardiac T2* values and spleen sizes were normally distributed. We used Spearman's rank-order method to seek correlations between the splenic $\mathrm{T} 2 *$ value and age, cardiac and hepatic $\mathrm{T} 2 *$ values, liver and spleen sizes, and serum ferritin levels. The Mann-Whitney $\mathrm{U}$ test was used to compare cardiac and hepatic T2* values, liver sizes and serum ferritin levels between patients who had and had not undergone splenectomies.

\section{Results}

The 131 patients had a mean age of 12.8 years (range: 4-34 years). There were 39 patients who had undergone splenectomies. The mean splenic $\mathrm{T} 2 \%$ value was 9.24 

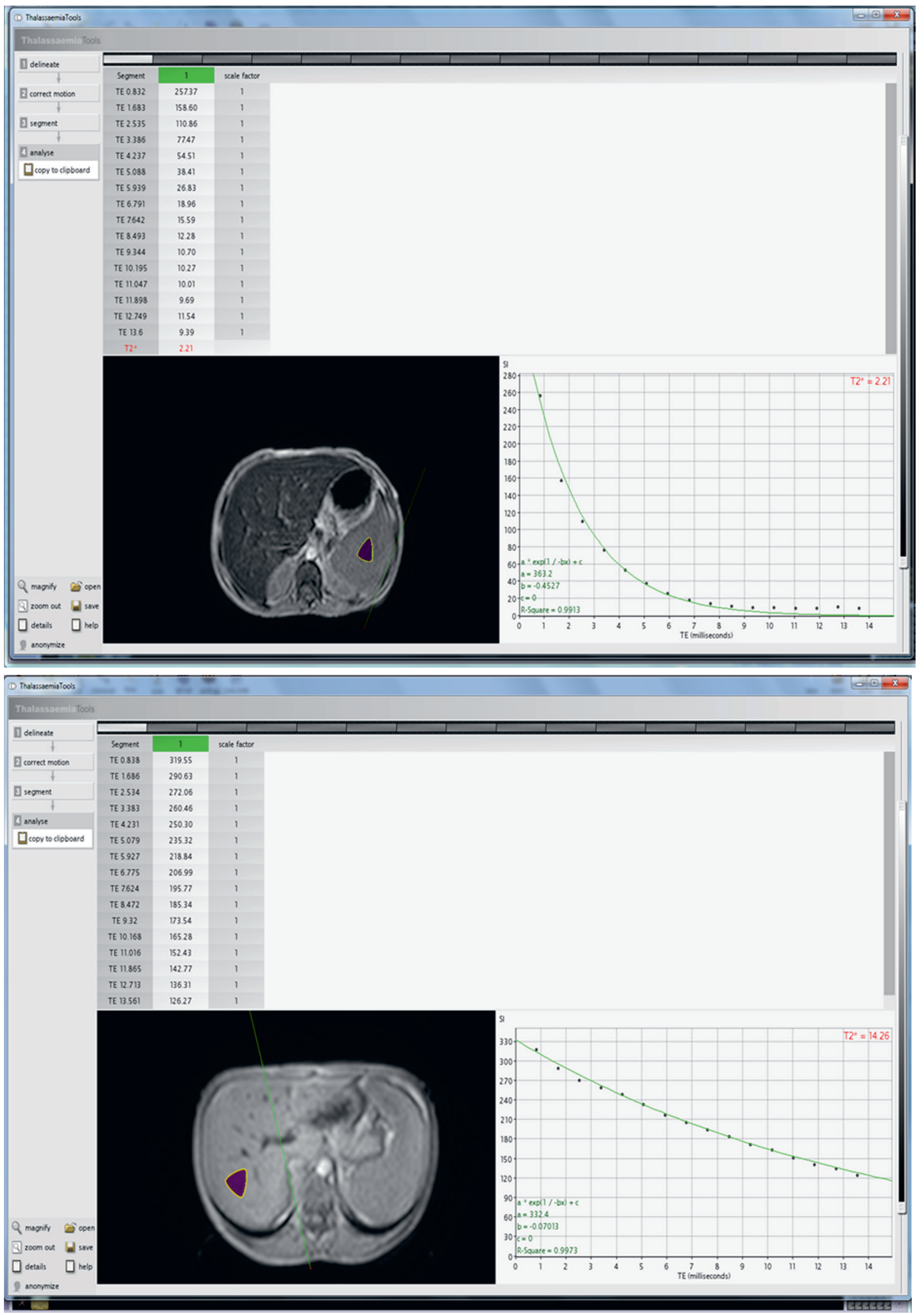

Fig. 1-2. Splenic and hepatic T2* assessments were performed on the central liver zones using a transverse plane multiecho gradient echo sequence. Region of interest (ROI) areas covered only tissue exhibiting no motion artifacts outside of vascular and subcapsular spaces 


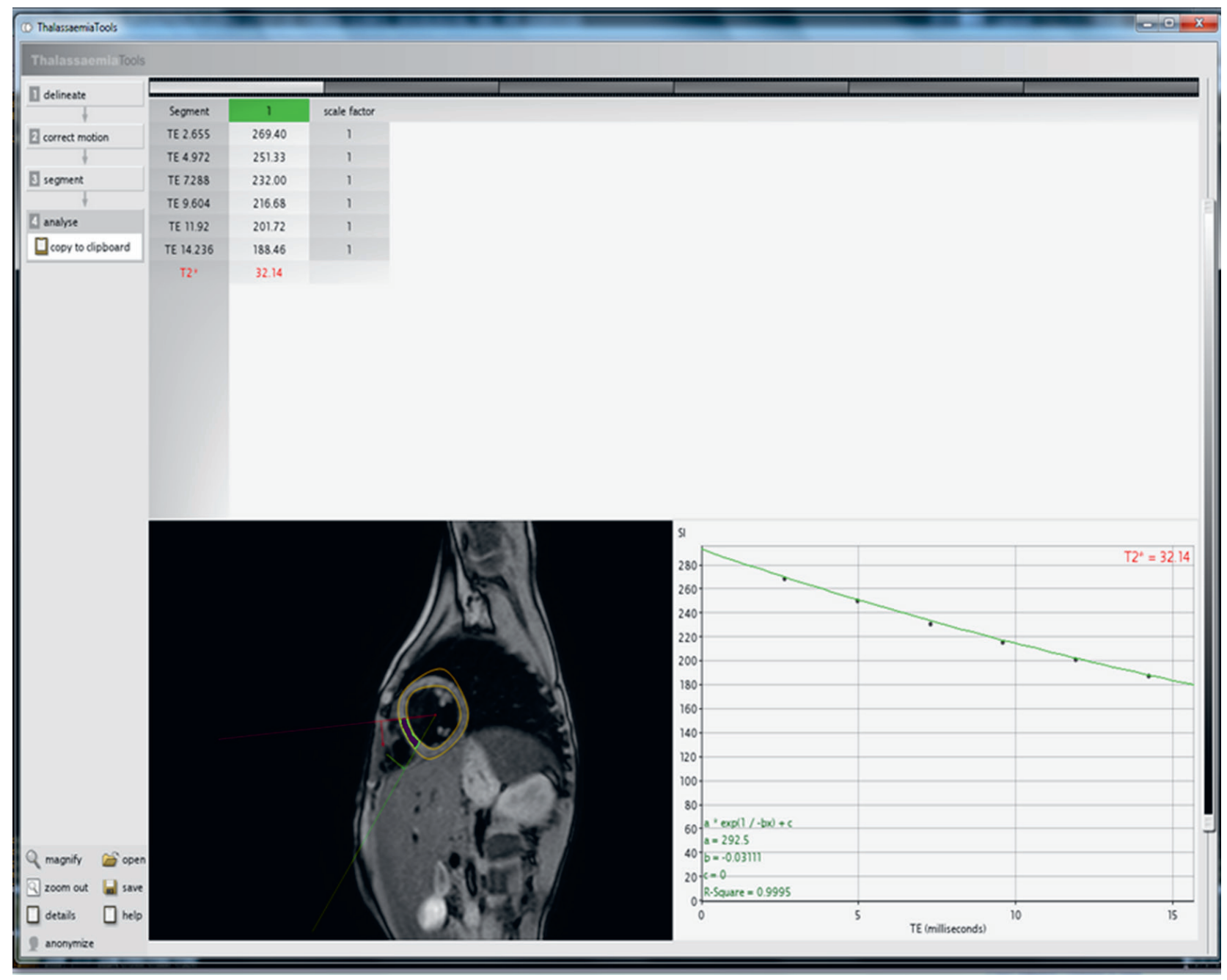

Fig. 3. Cardiac T2* assessments were performed on the ventricular septum using a short-axis, midventricular, cardiac-gated multiecho gradient echo sequence

(range: $0.75-48.75$ ) in the 92 patients who had not undergone splenectomies. Splenic T2* values correlated moderately with hepatic T2* values $(\mathrm{p}<0.001, \mathrm{r}=0.516)$ (Fig. 5). Serum ferritin levels correlated strongly with both splenic and hepatic T2* values $(\mathrm{p}<0.001$ and $0.001 ; \mathrm{r}=-0.728$ and -0.707 , respectively). Splenic $\mathrm{T} 2 *$ values correlated weakly with liver size $(\mathrm{p}=0.014)$, but not with age, cardiac or muscle $\mathrm{T} 2 *$ values, or spleen size $(\mathrm{p}=0.27,0.21,0.99$, and

Table 2. Relationships among splenic T2* values, liver and heart features, and serum ferritin levels

\begin{tabular}{|l|c|c|c|c|}
\multicolumn{1}{c|}{$\begin{array}{c}\text { Tissue } \\
\text { characteristics } \\
\text { and serum ferritin }\end{array}$} & Median (min-max) & p-value & r-value & $n$ \\
\hline Splenic size $[\mathrm{mm}]$ & $122(68-230)$ & 0.393 & -0.9 & 92 \\
\hline Liver T2* [ms] & $2.37(0.6-28.54)$ & $<0.001$ & 0.516 & 92 \\
\hline Liver size [mm] & $145(98-206)$ & 0.14 & -0.255 & 92 \\
\hline Cardiac T2* [ms] & $27.13(3.95-51.45)$ & 0.211 & 0.132 & 92 \\
\hline Muscle T2* [ms] & $28.14(13.93-38.68)$ & 0.992 & 0.001 & 73 \\
\hline Serum ferritin & $1,969(302-19,030)$ & $<0.001$ & -0.728 & 55 \\
\hline
\end{tabular}

$\mathrm{n}$ - number of patients.

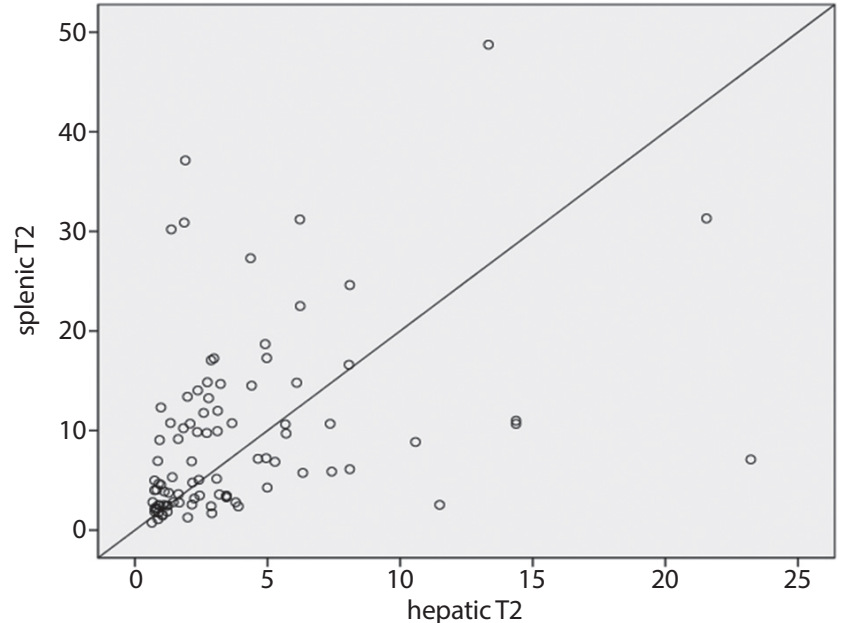

Fig. 5. Scatterplot of the positive correlation between splenic T2* and hepatic T2* values

0.39 , respectively) (Table 2 ). Muscle T2* values correlated with serum ferritin levels $(\mathrm{p}=0.022, \mathrm{r}=-297$ ) (Table 2).

Cardiac $\mathrm{T} 2 \%$ values were lower and liver sizes greater in patients who had undergone splenectomy $(\mathrm{p}<0.001$ 


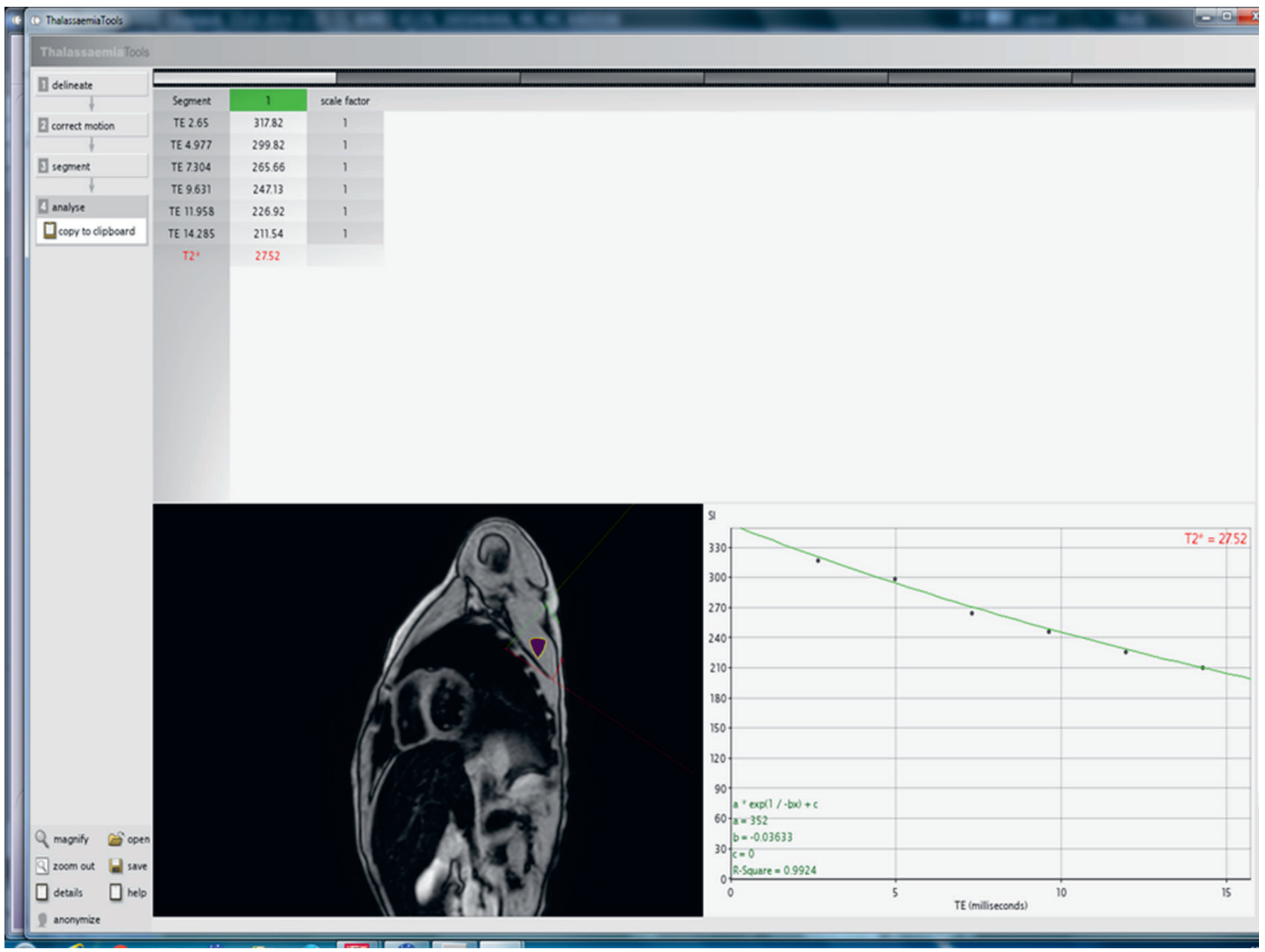

Fig. 4. T2* assessments of the left shoulder girdle muscles were done using short-axis cardiac sequences

Table 3. Comparison of iron loads and serum ferritin levels in patients who had and had not undergone splenectomies

\begin{tabular}{|l|c|c|c|c|c|}
\multirow{2}{*}{$\begin{array}{c}\text { Tissue } \\
\text { characteristics } \\
\text { and serum ferritin }\end{array}$} & \multicolumn{2}{c|}{$\begin{array}{c}\text { Patients with } \\
\text { splenectomy }\end{array}$} & \multicolumn{2}{c|}{$\begin{array}{c}\text { Patients without } \\
\text { splenectomy }\end{array}$} & \multirow{2}{*}{ p-value } \\
\cline { 2 - 5 } & median & $n$ & median & $n$ & \\
\hline Liver T2* [ms] & 1.86 & 37 & 2.42 & 92 & 0.421 \\
\hline Liver size [mm] & 161 & 39 & 141 & 92 & 0.001 \\
\hline Cardiac T2* [ms] & 22.27 & 39 & 29.45 & 92 & $<0.001$ \\
\hline Muscle T2* [ms] & 28.15 & 30 & 28.13 & 73 & 0.89 \\
\hline Serum ferritin & 2,800 & 18 & 1,966 & 37 & 0.468 \\
\hline
\end{tabular}

$n-$ number of patients.

and 0.001 , respectively). However, no significant betweengroup differences were evident in terms of muscle or hepatic $\mathrm{T} 2 \%$ values or serum ferritin level $(\mathrm{p}=0.85,0.42$ and 0.47 , respectively) (Table 3 ).

\section{Discussion}

Iron is an essential element involved in oxidative processes and is present in hemoglobin, myoglobin and many enzymes. The iron balance is regulated by increasing or reducing iron absorption. Humans cannot excrete excess iron. In TM patients, the total body iron level increases upon repeated transfusions in association with enhanced intestinal absorption. ${ }^{1,15}$ Moreover, intestinal iron absorption is accelerated by ineffective erythropoiesis and hypoxia. Senescent erythrocytes from transfused blood and cells resulting from ineffective erythropoiesis are digested by the Kupffer cells of the spleen and liver, ${ }^{16,17}$ increasing iron loading and the blood level of non-transferrin bound iron (NTBI). Non-transferrin bound iron triggers iron poisoning. High levels of NTBI often trigger iron accumulation in various organs, particularly the heart, pancreas and pituitary gland. Iron-mediated cardiac toxicity is a major cause of heart failure and death, particularly in TM patients. ${ }^{9,18,19}$ Excess iron in the pituitary gland and other organs triggers major complications including diabetes mellitus and hypogonadism. ${ }^{20}$

We found strong correlations between splenic $\mathrm{T} 2 \%$ and hepatic T2* values and serum ferritin levels, attributable to the presence of Kupffer cells (phagocytosing erythrocytes) in both the liver and spleen. Few prior studies have sought these correlations. Papakonstantinou et al. used $\mathrm{T} 2 \%$-weighted multiecho gradient-echo sequences to compare the signal intensity ratios of the spleen and 
liver to that of the right paraspinous muscle. No correlation was apparent, although all signal intensities correlated with the serum ferritin level. ${ }^{11}$ Brewer et al. found a weak correlation between the extent of splenic and hepatic iron deposition in thalassemia patients. ${ }^{12}$ Our present findings differ, perhaps due to the fact that the mean patient ages in both of the studies cited (16.4 and 24.2 years, respectively) were higher than that of our patients (the mean age of patients who had not undergone splenectomy was 11.4 years). Also, the study by Papakonstantinou et al. had a smaller patient series than ours. ${ }^{11}$

In our study a moderate correlation was apparent between hepatic and cardiac T2* values, but not between splenic and cardiac T2\% values. Excessive liver iron overload reduces the accuracy of $\mathrm{T} 2 \%$ imaging, attributable to signal constriction and loss. Although the T2* value is a strong indicator of hepatic iron overload, it does not correlate with liver iron concentrations..$^{1721,22}$ We suggest that the extent of splenic iron overload may be similar to that of the liver.

Splenectomy may have various effects on tissues. We found that although the cardiac $\mathrm{T} 2$ * value was lower in patients who had undergone splenectomy, no significant between-group differences were evident in terms of muscle $\mathrm{T} 2 *$ values. This indicated that cardiac iron overload increases after a splenectomy, because cardiac energy metabolism is faster than muscle energy metabolism. Moreover, the muscle $\mathrm{T} 2 *$ value correlated only weakly with the serum ferritin level, indicating that iron accumulation by muscles is low. Liver size was increased in patients who had undergone splenectomy, perhaps due to extramedullary hematopoiesis and a response to the iron overload. The spleen and liver are the primary iron deposition tissues in patients with thalassemia and play important roles in protection against increased iron levels. ${ }^{11,21}$ The spleen also eliminates old and defective erythrocytes. However, this function is performed by the liver in patients who have undergone splenectomy. To our knowledge, the study by Brewer et al. is the only work to explore iron overload in patients who have undergone splenectomy. Cardiac iron overload increased in 40 such patients, but the total hepatic iron load did not. ${ }^{12}$

The limitations of our study include the fact that hepatic iron overload was not confirmed by biopsy and we had no control group.

\section{Conclusions}

We found that splenic iron overload was strongly correlated with hepatic iron overload and the serum ferritin level. Splenectomy increased cardiac iron overload and liver size in TM patients. However, as the iron overload in muscles was low, this tissue was not affected by splenectomy.

\section{References}

1. Ganz T. Molecular control of iron transport. J Am SocNephrol. 2007; 18(2):394-400.

2. Morgan EH, Walters MNI. Iron storage in human disease. J Clin Pathol. 1963;16(2):101-107.

3. Rodeghiero F, Ruggeri M. Short- and long-term risks of splenectomy for benign haematological disorders: Should we revisit the indications? Br J Haematol. 2012;158(1):16-29.

4. Garbowski MW, Carpenter JP, Smith G, et al. Biopsy-based calibration of $\mathrm{T}^{*}$ magnetic resonance for estimation of liver iron concentration and comparison with R2 Ferriscan. J Cardiovasc Magn Reson. 2014;16:40.

5. Hankins JS, McCarville MB, Loeffler RB, et al. R2* magnetic resonance imaging of the liver in patients with iron overload. Blood. 2009; 113(20):4853-4855.

6. Wood JC. Guidelines for quantifying iron overload. ASHEduc Program Book. 2014;2014(1):210-215.

7. Christoforidis A, Haritandi A, Tsatra I, Tsitourides I, Karyda S, Athanassiou-Metaxa M. Four-year evaluation of myocardial and liver iron assessed prospectively with serial MRI scans in young patients with $\beta$-thalassaemia major: Comparison between different chelation regimens. Eur J Haematol. 2007;78(1):52-57.

8. Noetzli LJ, Carson SM, Nord AS, Coates TD, Wood JC. Longitudinal analysis of heart and liver iron in thalassemia major. Blood. 2008; 112(7):2973-2978.

9. Carpenter JP, Roughton M, Pennell DJ. International survey of T2* cardiovascular magnetic resonance in $\beta$-thalassemia major. Haematologica. 2013;98(9):1368-1374.

10. Wang ZJ, Fischer R, Chu Z, et al. Assessment of cardiac iron by MRI susceptometry and R2* in patients with thalassemia. Magn Reson Imaging. 2010;28(3):363-371.

11. Papakonstantinou O, Drakonaki EE, Maris T, Vasiliadou A, Papadakis A, Gourtsoyiannis N. MR imaging of spleen in beta-thalassemia major. Abdom Imaging. 2015;40(7):2777-2782.

12. Brewer CJ, Coates TD, Wood JC. Spleen R2 and R2* in iron-overloaded patients with sickle cell disease and thalassemia major. JMRI. 2009; 29(2):357-364.

13. Kolnagou A, Natsiopoulos K, Kleanthous M, loannou A, Kontoghiorghes GJ. Liver iron and serum ferritin levels are misleading for estimating cardiac, pancreatic, splenic and total body iron load in thalassemia patients: Factors influencing the heterogenic distribution of excess storage iron in organs as identified by MRI T2*. Toxicol Mech Methods. 2013;23(1):48-56.

14. Papakonstantinou O, Alexopoulou E, Economopoulos N, et al. Assessment of iron distribution between liver, spleen, pancreas, bone marrow, and myocardium by means of R2 relaxometry with MRI in patients with beta-thalassemia major. JMagn Reson Imaging. 2009; 29(4):853-859.

15. Tziomalos K, Perifanis V. Liver iron content determination by magnetic resonance imaging. WJG. 2010;16(13):1587-1597.

16. Siegelman ES, Mitchell DG Semelka RC. Abdominal iron deposition: Metabolism, MR findings, and clinical importance. Radiology. 1996; 199(1):13-22.

17. Gandon Y, Olivie D, Guyader D, et al. Non-invasive assessment of hepatic iron stores by MRI. The Lancet. 2004;363(9406):357-362.

18. Wood JC. Estimating tissue iron burden: Current status and future prospects. Br J Haematol. 2015;170(1):15-28.

19. Matter RM, Allam KE, Sadony AM. Gradient-echo magnetic resonance imaging study of pancreatic iron overload in young Egyptian beta-thalassemia major patients and effect of splenectomy. Diabetol Metab Syndr. 2010;2:23.

20. Galanello R, Origa R. Beta-thalassemia. Orphanet J Rare Dis. 2010;5:11.

21. Queiroz-Andrade $M$, Blasbalg R, Ortega $C D$, et al. MR imaging findings of iron overload. Radiogr Rev Publ Radiol Soc N Am Inc. 2009;29(6): 1575-1589.

22. Westphalen ACA, Qayyum A, Yeh BM, et al. Liver fat: Effect of hepatic iron deposition on evaluation with opposed-phase MR imaging. Radiology. 2007;242(2):450-455. 